\title{
Arctiin regulates collagen type $1 \alpha$ chain 1 mRNA expression in human dermal fibroblasts via the miR-378b-SIRT6 axis
}

\author{
KARAM KIM $^{1 *}$, SUNGKWAN AN ${ }^{2 *}$, BYUNG GON CHOI $^{3}$, DAHYE JOO ${ }^{1}$, \\ YOUNG MIN CHOI ${ }^{1}, \mathrm{KYU}_{\text {JOONG AHN }}^{3}$, IN-SOOK AN ${ }^{1}$ and HWA JUN CHA ${ }^{2,4}$
}

${ }^{1}$ KISCS Incorporated, 2nd Enterprise Research Building, Cheongju, Chungcheongbuk 361-951; ${ }^{2}$ Korea Institute for Skin and Clinical Sciences, Konkuk University; ${ }^{3}$ Department of Dermatology, Konkuk University School of Medicine, Seoul 143-701; ${ }^{4}$ Department of Skin Care \& Beauty, Osan University, Osan, Gyeonggi-do 18119, Republic of South Korea

Received February 28, 2016; Accepted February 10, 2017

DOI: $10.3892 / \mathrm{mmr} .2017 .7679$

\begin{abstract}
Arctiin, a lignin isolated from Arctium lappa, exhibits a variety of biological effects, including anti-viral, anti-inflammatory, and anti-proliferative actions, in mammalian cells. In a previous study, arctiin was demonstrated to induce procollagen type I synthesis and exhibited protective effects against ultraviolet $B$ (UVB) radiation in normal human dermal fibroblasts (nHDFs). However, the underlying molecular mechanism of arctiin-mediated collagen synthesis remains unknown. In the present study, the mechanism for increased expression of collagen type $1 \alpha 1$ chain (COL1A1) mRNA in arctiin-induced nHDFs was identified. The expression of microRNA-378b (miR-378b), downregulated by arctiin, was correlated with the expression of sirtuin-6 (SIRT6) mRNA, a regulator of COL1A1 mRNA. Furthermore, it was revealed that arctiin protected the UVB radiation-mediated decrease in COL1A1 mRNA expression, through the miR-378b/SIRT6 signaling pathway. In conclusion, these results suggest that arctiin regulates COL1A1 through the miR-378b-SIRT6 axis.
\end{abstract}

\section{Introduction}

The human skin surrounds the external surface of the human body and acts as a protector against environmental factors, including ultraviolet (UV) irradiation, destructive agents and viral infections $(1,2)$. The skin consists of two layers, the epidermis and dermis. The epidermis forms an epidermal barrier through keratinocyte differentiation (3). The dermis consists of an extracellular matrix that is predominantly composed of type I collagen synthesized by fibroblasts (4-6).

Correspondence to: Dr Hwa Jun Cha, Korea Institute for Skin and Clinical Sciences, Konkuk University, 120 Neungdong Road, Seoul 143-701, Republic of South Korea

E-mail: hjcha@konkuk.ac.kr

${ }^{*}$ Contributed equally

Key words: microRNA-378b, microRNA, collagen, sirtuin-6, arctiin
The skin loses these two functions as it ages, due to intrinsic aging and extrinsic factors (7). Intrinsic factor-induced skin aging is a natural skin aging process, caused by the loss of function of age-associated genes due to heredity or the passage of time (8). Extrinsic factor-induced skin aging is aging or photoaging, caused by environmental conditions, predominantly from exposure to UV radiation (9). UV-induced oxidative stress is accelerated by deep wrinkles caused by the loss of collagen production $(10,11)$. Therefore, an endogenous anti-oxidant defense system is required for protection against the effects of photodamage, which contribute to skin aging (12).

Arctiin, a lignin compound isolated from Arctium lappa, also known as greater burdock, possesses a variety of anti-viral, anti-inflammatory and anti-proliferative effects in mammalian cells (13-15). Arctiin has recently been demonstrated to improve procollagen type I synthesis and exhibits a protective effect against ultraviolet B (UVB) radiation $(16,17)$. An extract of A. lappa or isolated arctiin induces collagen synthesis in the dermis (18). However, the underlying mechanism of collagen synthesis, induced by arctiin treatment, remains unknown.

MicroRNAs (miRNAs) are small non-coding RNAs that function as important regulators of gene expression in skin aging and differentiation (19-22). These miRNAs are 16-35 nucleotides (nts) in length (median, 21-22 nts) that interact with the $3^{\prime}$ untranslated region (3' UTR) of target messenger RNAs (mRNAs). Consequently, the complementary interaction of miRNA and its target mRNA inhibits protein translation at the post-transcriptional level $(23,24)$. In skin aging, these miRNAs serve a key role by targeting skin aging-associated gene expression and UV protective-associated gene expression in the dermis (25-27). A previous study revealed that miRNAs serve an important role in anti-aging functions and skin stress responses of skin-derived cells, including keratinocytes and dermal fibroblasts (28). Additionally, several miRNAs have been reported to regulate melanogenesis, skin aging, and differentiation of melanocytes, keratinocytes, and dermal fibroblasts $(29,30)$.

In a previous study, the authors revealed that arctiin inhibited microRNA-378b (miR-378b) expression in UVB-irradiated normal human dermal fibroblasts (nHDFs) (16). Therefore, in the present study, it was investigated whether this alteration of 
A

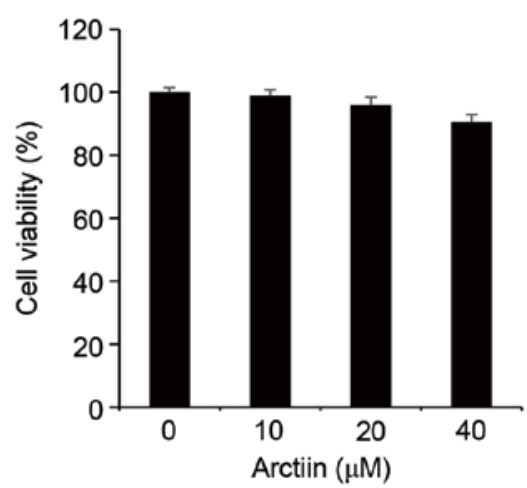

B

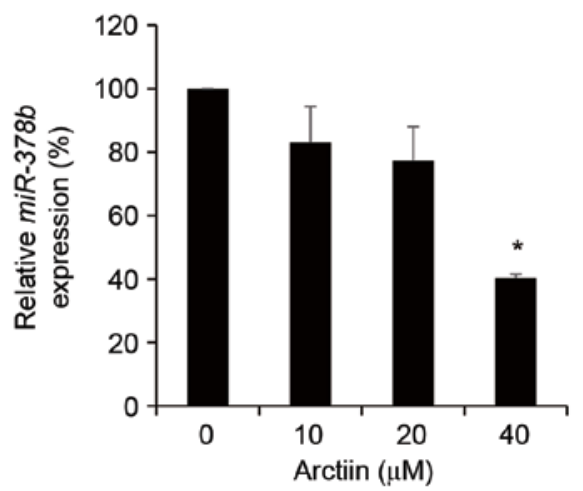

Figure 1. Arctiin decreases miR-378b expression in nHDFs. (A) Arctiin causes no significant cytotoxicity in nHDFs. Cells were seeded in 96-well culture plates and treated with the indicated doses of arctiin $(0,10,20$ or $40 \mu \mathrm{M})$. After $24 \mathrm{~h}$ of incubation, a water-soluble tetrazolium salt-based cytotoxicity assay was performed. Each bar represents the mean + standard deviation from three independent experiments. (B) Analysis of relative miR-378b expression in arctiin-treated nHDFs. Cells were treated with the indicated doses of arctiin $(0,10,20$, or $40 \mu \mathrm{M})$ for $24 \mathrm{~h}$. The level of miR-378b expression was determined using the reverse transcription-quantitative polymerase chain reaction. Results were normalized to the U6 small nuclear RNA expression level. The data are representative of three independent experiments. ${ }^{*} \mathrm{P}<0.05$ vs. control. miR-378b, microRNA-378b; nHDFs, normal human dermal fibroblasts.

miR-378b contributes to the enhanced procollagen synthesis induced by treatment with arctiin in nHDFs. A detailed mechanism by which arctiin stimulates collagen synthesis in nHDFs, by inhibiting miR-378b expression that, in turn, increases expression of its target sirtuin-6 (SIRT6) through reduced miRNA-mediated repression is demonstrated.

\section{Materials and methods}

Cell culture, chemicals, and reagents. nHDFs (Lonza Group, Ltd., Basel, Switzerland) were cultured in Dulbecco's Modified Eagle medium containing 10\% fetal bovine serum (both HyClone; GE Healthcare Life Sciences, Logan, UT, USA) and antibiotics in a humidified incubator under $5 \% \mathrm{CO}_{2}$ at $37^{\circ} \mathrm{C}$. Arctiin was purchased from Sigma-Aldrich (Merck KGaA, Darmstadt, Germany) and dissolved in dimethyl sulfoxide.

Arctiin treatment and $U V B$ exposure. For arctiin treatment alone, $\mathrm{nHDFs}\left(1 \times 10^{5}\right.$ cells in $60 \mathrm{~mm}$ culture dish and $3 \times 10^{3}$ cells in 96-well plates) were incubated with arctiin at $37^{\circ} \mathrm{C}$ for $24 \mathrm{~h}$, and then a cell viability assay and reverse transcription-quantitative polymerase chain reaction were performed. At UVB exposure, $\mathrm{nHDF}$ ( $1 \times 10^{5}$ cells in $60 \mathrm{~mm}$ culture dish and $3 \times 10^{3}$ cells in 96-well plates) was pre-incubated with arctiin at $37^{\circ} \mathrm{C}$ for $3 \mathrm{~h}$, and then $30 \mathrm{~mJ} / \mathrm{cm}^{2} \mathrm{UVB}$ was exposed to arctiin-pretreated nHDFs. After UVB exposure, the nHDFs were incubated at $37^{\circ} \mathrm{C}$ for $24 \mathrm{~h}$ and then western blotting and RT-qPCR were performed.

Cell viability assay. The cytotoxic effects of arctiin on nHDFs were determined using a water-soluble tetrazolium salt (WST-1) assay (EZ-Cytox cell viability assay kit; ITS Bio, Seoul, Korea). nHDFs were seeded at a density of $3 \times 10^{3}$ cells in 96 -well plates and incubated for $24 \mathrm{~h}$. The cells were then incubated with $0-40 \mu \mathrm{M}$ arctiin for $24 \mathrm{~h}$. The WST-1 assay solution was added to the cells at a $1 / 10$ volume of the total culture medium and were incubated at $37^{\circ} \mathrm{C}$ for $1 \mathrm{~h}$. Cell viability was evaluated by measuring the absorbance at $450 \mathrm{~nm}$, using an iMark microplate reader (Bio-Rad Laboratories, Inc., Hercules, CA, USA).

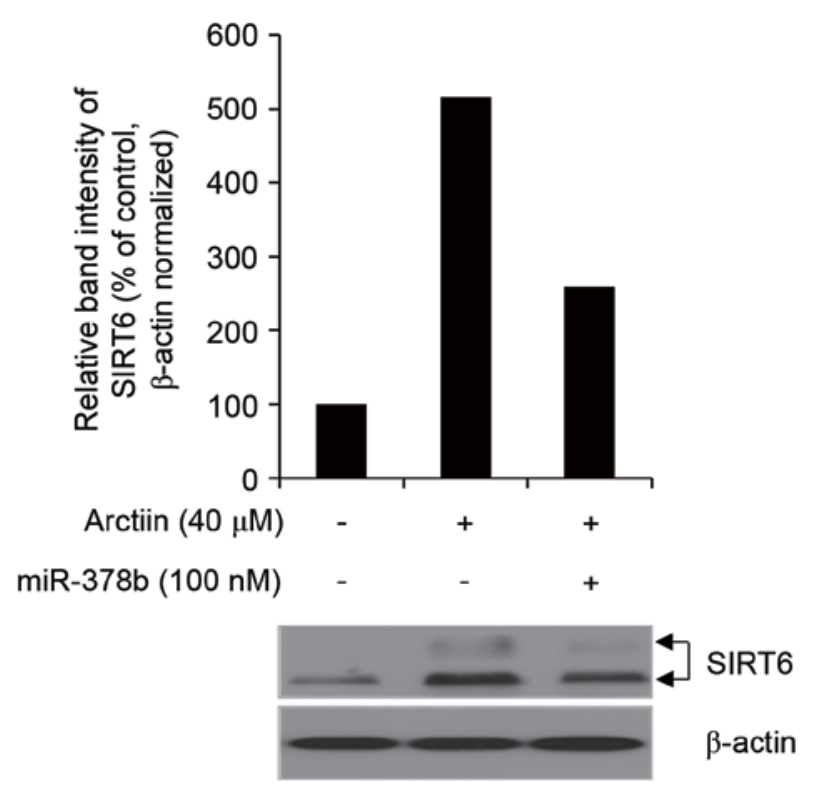

Figure 2. Arctiin increases SIRT6 protein expression in nHDFs through miR-378b. Western blot analysis of SIRT6 expression in nHDFs transfected with miR-378b or negative control miRNA and incubated in the presence or absence of arctiin $(40 \mu \mathrm{M})$. After $24 \mathrm{~h}$ of incubation, the levels of SIRT6 proteins were evaluated by western blotting analysis. $\beta$-actin was used as an internal control. Sirtuin-6, SIRT6; nHDFs, normal human dermal fibroblasts; microRNA-378b, miR-378b.

Transfection of a miR-378b mimic and anti-miR-378b. An $m i R-378 b$ mimic (100 nM; 5'-ACUGGACUUGGA GGCAGAA-3'), an anti-miR-378b (100 nM; 5'-ACUGGA CUUGGAGGCAGAA-3'), and $100 \mathrm{nM}$ scrambled control (AccuTarget $^{\mathrm{TM}}$ negative control siRNA; Bioneer Corporation, Daejeon, Korea) were purchased from Qiagen $\mathrm{GmbH}$ (Hilden, Germany). miRNAs were dissolved in nuclease-free water (USB Biochemicals; Affymetrix, Inc., Santa Clara, CA, USA). nHDFs were seeded at a density of $1 \times 10^{5}$ cells in $60 \mathrm{~mm}$ culture dish and incubated for $24 \mathrm{~h}$. Cells were transfected with the miRNA mimic, anti-miRNA of $m i R-378 b$, or the negative control using Lipofectamine RNAiMAX (Invitrogen; Thermo 
A

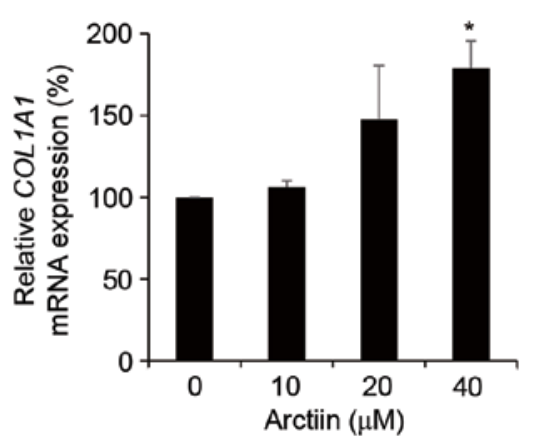

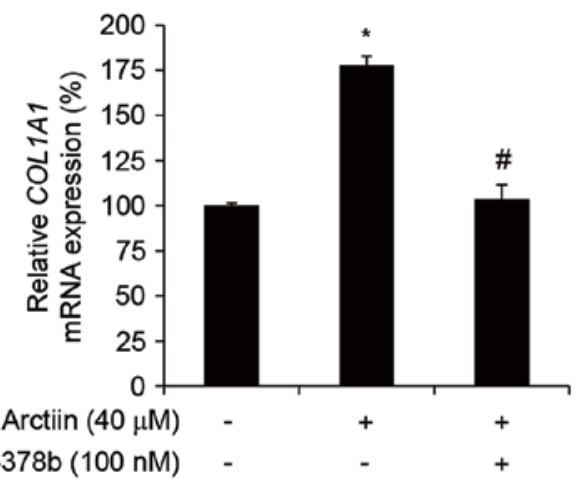

Figure 3. Arctiin increases COL1A1 mRNA expression in nHDFs through inhibition of miR-378b. (A) Analysis of relative COL1A1 mRNA expression in arctiin-treated nHDFs. Cells were treated with the indicated doses of arctiin $(0,10,20$, or $40 \mu \mathrm{M})$ for $24 \mathrm{~h}$. The level of COL1A1 mRNA was determined by RT-qPCR. (B) Analysis of miR-378b-mediated COL1A1 mRNA expression in arctiin-treated nHDFs. After a 24-h incubation, the level of COL1A1 mRNA was determined by RT-qPCR. Values represent the mean + standard deviation of three independent experiments. ${ }^{*} \mathrm{P}<0.05$ vs. control; ${ }^{*} \mathrm{P}<0.05$ vs. arctiin-treated nHDFs. nHDFs, normal human dermal fibroblasts; COL1A1, collagen type $1 \alpha$ chain 1; miR-378b, microRNA-378b; RT-qPCR, reverse transcription-quantitative polymerase chain reaction.

Fisher Scientific, Inc., Waltham, MA, USA) according to the manufacturer's protocol.

$R N A$ preparation and $R T-q P C R$. Total RNA was isolated using TRIzol reagent (Invitrogen; Thermo Fisher Scientific, Inc.) according to the manufacturer's protocol. The purity and concentration of the RNA samples were estimated using MaestroNano ${ }^{\circledR}$ (Maestrogen, Inc., Las Vegas, NV, USA). cDNA was synthesised from total RNA using the SuperScript ${ }^{\mathrm{TM}}$ III Reverse Transcriptase kit (Invitrogen; Thermo Fisher Scientific, Inc.) according to the manufacturer's protocol. To evaluate collagen type $1^{\alpha} 1$ chain (COL1A1) mRNA expression, qPCR was performed using COL1A1 primers (human COL1A1 forward: 5'-AGGGCCAAGACATC-3'; and reverse: 5'-AGATCACGTCATCGCACAACA-3') and EvaGreen premix (Solis BioDyne, Tartu, Estonia) with the StepOnePlus ${ }^{\mathrm{TM}}$ real-time PCR system (Applied Biosystems; Thermo Fisher Scientific, Inc., Waltham, MA, USA). Expression was normalized to $\beta$-actin (human $\beta$-actin forward: 5'-GGATTCCTA TGTGGGCGACGA-3' and reverse: 5'-GCTCGGTGAGGA TCTTCATG-3'). Additionally, cDNAs for miR-378b detection were synthesized using the miScript II RT Kit (Qiagen Inc., Valencia, CA, USA) according to the manufacturer's protocol. qPCR was performed using a miR-378b-specific primer, Hs miR-1290_1 miScript Primer Assay (Qiagen Inc., Valencia, CA, USA), and the miScript SYBR-Green PCR Kit (Qiagen Inc., Valencia, CA, USA) with the StepOnePlus ${ }^{\mathrm{TM}}$ real-time PCR system. MiR-378b expression was normalized to U6 small nuclear RNA. The $2^{-\Delta \Delta \mathrm{Cq}}$ method was used to calculate the relative expression level of COL1A1 and miR-378b (31). Cycling conditions were as follows: An initial predenaturation step at $94^{\circ} \mathrm{C}$ for $5 \mathrm{~min}$, followed by 40 cycles of denaturation at $94^{\circ} \mathrm{C}$ for $30 \mathrm{sec}$, annealing at $60^{\circ} \mathrm{C}$ for $30 \mathrm{sec}$, extension at $72^{\circ} \mathrm{C}$ for $30 \mathrm{sec}$ and a final extension step at $72^{\circ} \mathrm{C}$ for $5 \mathrm{~min}$. All experiments were repeated three times. Data was analysed using Microsoft Excel 2016 (Microsoft Corporation, Redmond, WA, USA) and presented as the mean \pm standard deviation.

Western blot analysis. Cells were analysed using radioimmunoprecipitation assay buffer $(50 \mathrm{mM}$ Tris- $\mathrm{Cl} \mathrm{pH}=8.0,150 \mathrm{mM}$

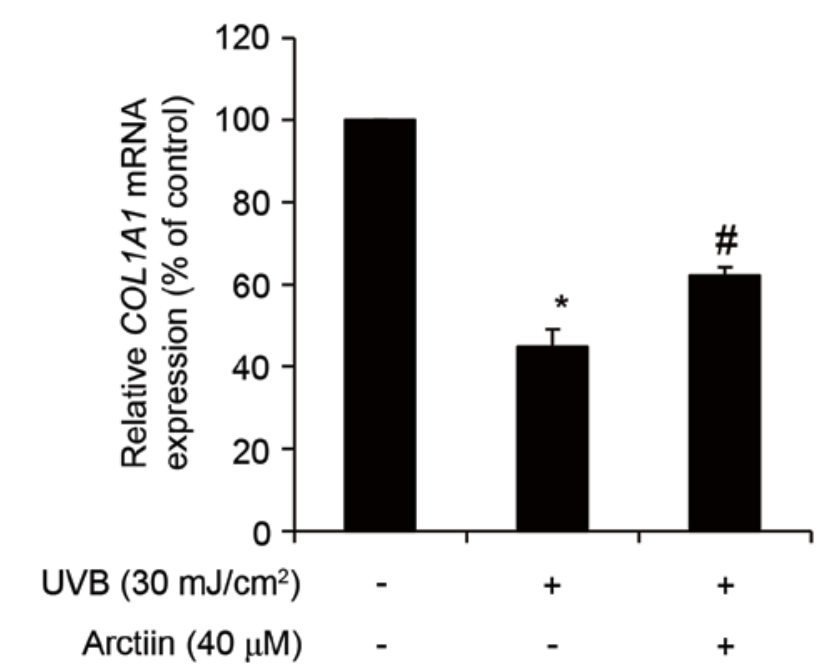

Figure 4. Arctiin attenuates UVB-mediated decrease in COL1A1 mRNA in nHDFs. nHDFs were pretreated with arctiin for $6 \mathrm{~h}$ and then exposed to $30 \mathrm{~mJ} / \mathrm{cm}^{2}$ UVB. After $24 \mathrm{~h}$, COL1A1 mRNA expression was determined by the reverse transcription-polymerase chain reaction. Values represent the mean + standard deviation of three independent experiments. ${ }^{*} \mathrm{P}<0.05$ vs. controls; "P<0.05 vs. UVB-treated nHDFs. UVB, ultraviolet B; COL1A1, collagen type $1 \alpha$ chain 1 chain; nHDFs, normal human dermal fibroblasts.

$\mathrm{NaCl}, 1 \% \mathrm{NP}-40,0.5 \%$ sodium deoxycholate, and $0.1 \%$ SDS) containing protease inhibitors (EASYpack; Roche Applied Science, Mannheim, Germany). The protein concentration was measured using the Bradford assay (Bio-Rad Laboratories, Inc.). A total of $50 \mu \mathrm{g}$ cellular protein was dissolved in SDS sample buffer. Proteins were separated by SDS-PAGE on a $10 \%$ gel and transferred to polyvinylidene difluoride membranes (EMD Millipore, Billerica, MA, USA). The membranes were blocked in 5\% skimmed milk buffer (in $50 \mathrm{mM}$ Tris, $150 \mathrm{mM} \mathrm{NaCl}$ and $0.1 \%$ Tween 20) for $1 \mathrm{~h}$ at room temperature and were probed with rabbit anti-SIRT6 (1:2,000; catalog no. 12486; Cell Signalling Technology, Inc., Danvers, MA, USA) or anti- $\beta$-actin $(1: 10,000 ; \mathrm{N}-21$; catalog no. sc-130656; Santa Cruz Biotechnology, Inc., Dallas, TX, USA) primary antibodies at $4^{\circ} \mathrm{C}$ for $18 \mathrm{~h}$. Subsequently, these proteins were 
A

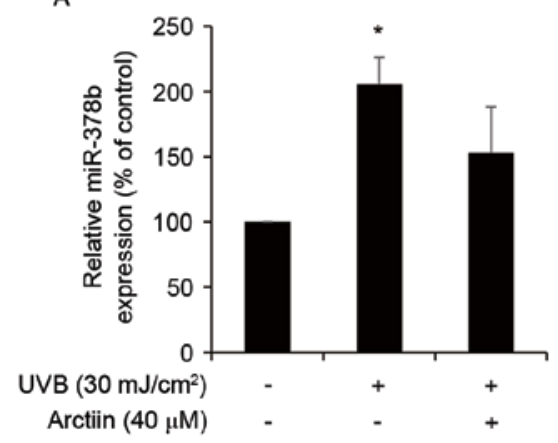

B

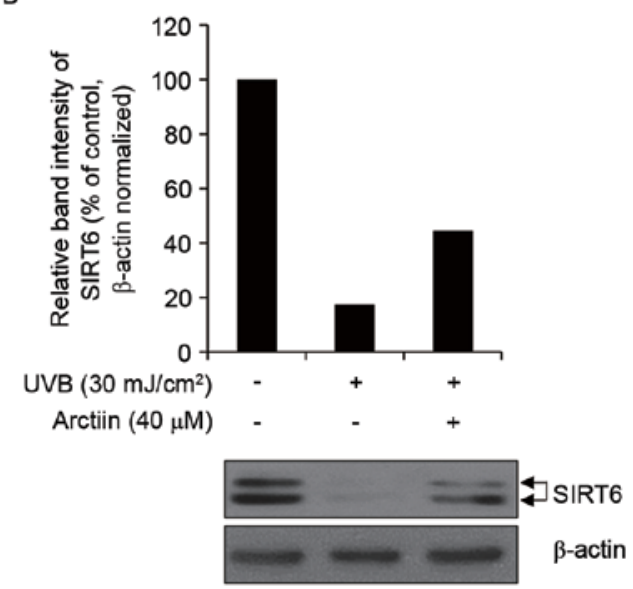

Figure 5. Arctiin regulates the miR-378b-SIRT6 axis in UVB-exposed nHDFs. (A) miR-378b expression was restored by arctiin in 30 mJ/cm ${ }^{2}$ UVB-exposed nHDFs. Cells were treated with UVB radiation either following the exposure to arctiin or in its absence. The level of miR-378b expression was determined by the reverse transcription-polymerase chain reaction. Results were normalized to U6 small nuclear RNA expression level. The data are representative of three independent experiments. ${ }^{*} \mathrm{P}<0.05$ vs. controls; ${ }^{*} \mathrm{P}<0.05$ vs. UVB-treated nHDFs. (B) SIRT6 protein expression was restored by treatment with arctiin in UVB-exposed nHDFs. Cells were treated with UVB radiation following either the presence or the absence of arctiin. The level of SIRT6 was determined by western blotting. $\beta$-actin was used as a protein loading control. nHDFs, normal human dermal fibroblasts; miR-378b, microRNA-378b; UVB, ultraviolet B; SIRT6, sirtuin-6.

visualized using an anti-mouse IgG horseradish peroxidase (HRP) (1:5,000; catalog no. 7076; Cell Signalling Technology, Inc.) and anti-rabbit IgG HRP (1;3,000; catalog no. 7074; Cell Signalling Technology, Inc.) secondary antibodies at $25^{\circ} \mathrm{C}$ for 1 h. A SuperSignal ${ }^{\mathrm{TM}}$ West Pico Chemiluminescent Substrate (Thermo Fisher Scientific, Inc.) and a ChemiDoc Touch Imaging system (Bio-Rad Laboratories) were used to visualise protein bands.

Statistical analysis. Statistical significance was calculated using one-way analysis of variance with Tukey's post-hoc test. $\mathrm{P}<0.05$ was considered to indicate a statistically significant difference using Microsoft Excel 2016 (Microsoft Corporation). Data are presented as the mean \pm standard error.

\section{Results and Discussion}

Arctiin reduces miR-378b expression in $n H D F s$. Arctiin has been reported to possess a variety of biological effects, including anti-viral, anti-inflammatory and anti-proliferative, in mammalian cells $(32,33)$. Binic et al $(34)$ revealed that arctiin induces collagen synthesis in $\mathrm{nHDF}$. These results have provided a novel insight into the anti-aging effect of arctiin on the skin. However, the underlying mechanism of collagen induction by treatment with arctiin remains unknown. In a previous study, it was revealed that arctiin markedly inhibited miR-378b in UVB-irradiated nHDFs (16). Therefore, in the present study, the miRNA-dependent mechanism with which COL1A1 is induced in arctiin-treated nHDFs was investigated.

Cell viability in nHDFs was not affected at a concentration of $0-40 \mu \mathrm{M}$ arctiin (Fig. 1A). Furthermore, in nHDFs, miR-378b levels decreased by treatment with arctiin in a dose-dependent manner (Fig. 1B). Treatment with $40 \mu \mathrm{M}$ arctiin significantly downregulated miR-378b by $40.34 \%$ compared with control cells $(\mathrm{P}<0.05)$. Therefore, in the subsequent experiments a dose of arctiin $<40 \mu \mathrm{M}$ was used.
Arctiin increases COL1A1 expression through the miR-378b-SIRT6 axis. Our ongoing study revealed that miR-378b repressed COL1A1 expression, interfering with SIRT6 expression (data unpublished). Therefore, it is hypothesized that miR-378b downregulates COL1A 1 indirectly by interfering with the translation of SIRT6 mRNA. Additionally, SIRT6 silencing is implicated in the regulation of COL1A1 expression and skin aging in human dermal fibroblasts (35). Treatment with $40 \mu \mathrm{M}$ of arctiin increased SIRT6 protein expression in nHDFs in Fig. 2, demonstrating that arctiin regulates SIRT6 in nHDFs. Furthermore, $m i R-378 b$ inhibited the arctiin-mediated increase in SIRT6 protein expression (Fig. 2). These results indicate that arctiin elevates SIRT6 protein expression indirectly, through inhibition of $m i R-378 b$. Furthermore, the arctiin-mediated increase of COL1A1 gene was antagonized by the addition of exogenous $m i R-378 b$ (Fig. 3B). Arctiin regulates COL1A1 via the $m i R-378 b$-SIRT6 axis.

Arctiin protects against UVB-induced decrease in COL1A1 levels in $n H D F s$. Previous studies have indicated that arctiin has protective effects against UVB radiation in nHDFs (16). It was examined whether this protective effect of arctiin against UVB opposed the photoaging-dependent decrease in COL1A1 levels in nHDFs. nHDFs were pretreated with $40 \mu \mathrm{M}$ arctiin for $6 \mathrm{~h}$ and then exposed to $100 \mathrm{~mJ} / \mathrm{cm}^{2} \mathrm{UVB}$ radiation. Pre-treatment with arctiin alleviated the UVB-induced inhibitory effects on COL1A1 mRNA expression (Fig. 4). Furthermore, UVB treatment increased miR-378b expression and decreased SIRT6 protein expression in nHDFs (Fig. 5A and B). Conversely, arctiin treatment decreased miR-378b expression and increased SIRT6 in UVB-exposed nHDFs. These inverse correlations indicated that arctiin elevates COL1A1 transcription indirectly in UVB-exposed nHDFs through loss of miR-378b-mediated inhibition of SIRT6. Alteration of Col1A1 expression, by enhanced SIRT6, is induced by repression of NF- $\mathrm{B}$ activation (35). In various aging mechanism, activation $N F-\kappa B$ signaling is a key 
mediator of aging $(36,37)$. In UVB-induced photoaging, NF- $\mathrm{KB}$ activity is implicated, since NF- $\kappa \mathrm{B}$ is indirectly and directly regulated by collagens and matrix metalloproteinases (38). Therefore, the results suggest that miR-378b is useful in preventing aging through blocking of SIRT6.

In conclusion, the study demonstrated that the arctiin-induced upregulation of COL1A1 expression is regulated by the miR-378/SIRT6 signaling pathway. Furthermore, it was revealed that arctiin is able to prevent the UVB-induced reduction of COL1A1 expression, at least partially, through this same pathway.

\section{Acknowledgements}

The present study was supported by the Research Professor Program of Konkuk University (to Professor Hwa Jun Cha) and the Korean Health Technology R\&D Project, Ministry of Health \& Welfare, Republic of Korea (grant no. HN13C0075).

\section{References}

1. Quan T and Fisher GJ: Role of age-associated alterations of the dermal extracellular matrix microenvironment in human skin aging: A mini-review. Gerontology 61: 427-434, 2015.

2. Darlenski R, Kazandjieva J and Tsankov N: Skin barrier function: Morphological basis and regulatory mechanisms. J Clin Med 4: 36-45, 2011.

3. Eckert RL and Rorke EA: Molecular biology of keratinocyte differentiation. Environ Health Perspect 80: 109-116, 1989.

4. Varani J, Dame MK, Rittie L, Fligiel SE, Kang S, Fisher GJ and Voorhees JJ: Decreased collagen production in chronologically aged skin: Roles of age-dependent alteration in fibroblast function and defective mechanical stimulation. Am J Pathol 168: 1861-1868, 2006.

5. Wulf HC, Sandby-Møller J, Kobayasi T and Gniadecki R: Skin aging and natural photoprotection. Micron 35: 185-191, 2004.

6. Egbert M, Ruetze M, Sattler M, Wenck H, Gallinat S, Lucius R and Weise JM: The matricellular protein periostin contributes to proper collagen function and is downregulated during skin aging. J Dermatol Sci 73: 40-48, 2014.

7. Farage MA, Miller KW, Elsner P and Maibach HI: Characteristics of the aging skin. Adv Wound Care (New Rochelle) 2: 5-10, 2013

8. Debacq-Chainiaux F, Leduc C, Verbeke A and Toussaint O: UV stress and aging. Dermatoendocrinol 4: 236-240, 2012.

9. Katiyar SK and Mukhtar H: Green tea polyphenol (-)-epigallocatechin-3-gallate treatment to mouse skin prevents UVB-induced infiltration of leukocytes, depletion of antigenpresenting cells, and oxidative stress. J Leukoc Biol 69: 719-726, 2001.

10. Wlaschek M, Tantcheva-Poór I, Naderi L, Ma W, Schneider LA, Razi-Wolf Z, Schüller J and Scharffetter-Kochanek K: Solar UV irradiation and dermal photoaging. J Photochem Photobiol B 63: 41-51, 2001.

11. Fisher GJ, Kang S, Varani J, Bata-Csorgo Z, Wan Y, Datta S and Voorhees JJ: Mechanisms of photoaging and chronological skin aging. Arch Dermatol 138: 1462-1470, 2002.

12. Shindo Y, Witt E and Packer L: Antioxidant defense mechanisms in murine epidermis and dermis and their responses to ultraviolet light. J Invest Dermatol 100: 260-265, 1993.

13. Lee S, Shin S, Kim H, Han S, Kim K, Kwon J, Kwak JH, Lee CK, Ha NJ, Yim D and Kim K: Anti-inflammatory function of arctiin by inhibiting COX-2 expression via NF- $\mathrm{B}$ pathways. J Inflamm (Lond) 8: 16, 2011

14. Wu JG, Wu JZ, Sun LN, Han T, Du J, Ye Q, Zhang H and Zhang YG: Ameliorative effects of arctiin from Arctium lappa on experimental glomerulonephritis in rats. Phytomedicine 16: 1033-1041, 2009

15. Knott A, Reuschlein K, Mielke H, Wensorra U, Mummert C, Koop U, Kausch M, Kolbe L, Peters N, Stäb F, et al: Natural Arctium lappa fruit extract improves the clinical signs of aging skin. J Cosmet Dermatol 7: 281-289, 2008.
16. Cha HJ, Lee GT, Lee KS, Lee KK, Hong JT, Lee NK, Kim SY, Lee BM, An IS, Hahn HJ, et al: Photoprotective effect of arctiin against ultraviolet $\mathrm{B}$-induced damage in $\mathrm{HaCaT}$ keratinocytes is mediated by microRNA expression changes. Mol Med Rep 10: 1363-1370, 2014

17. Syed DN, Khan MI, Shabbir M and Mukhtar H: MicroRNAs in skin response to UV radiation. Curr Drug Targets 14: 1128-1134, 2013.

18. Honda N,Jinnin M,Kajihara I, Makino T, MakinoK, Masuguchi S, Fukushima S, Okamoto Y, Hasegawa M, Fujimoto M and Ihn $\mathrm{H}$ : TGF- $\beta$-mediated down-regulation of microRNA-196a contributes to the constitutive upregulated type I collagen expression in scleroderma dermal fibroblasts. J Immunol 188: 3323-3331, 2012.

19. Yamauchi M, Prisayanh P, Haque Z and Woodley DT: Collagen cross-linking in sun-exposed and unexposed sites of aged human skin. J Invest Dermatol 97: 938-941, 1991.

20. Sohal RS and Weindruch R: Oxidative stress, caloric restriction, and aging. Science 273: 59-63, 1996.

21. Benedetto AV: The environment and skin aging. Clinics Derm 16: 129-139, 1998.

22. Kwon DN, Chang BS and Kim JH: MicroRNA dysregulation in liver and pancreas of CMP-Neu5Ac hydroxylase null mice disrupts insulin/PI3K-AKT signaling. Biomed Res Int 2014: 236385, 2014

23. Al-Gubory KH, Fowler PA and Garrel C: The roles of cellular reactive oxygen species, oxidative stress and antioxidants in pregnancy outcomes. Int J Biochem Cell Biol 42: 1634-1650, 2010.

24. Lucas K and Raikhel AS: Insect microRNAs: Biogenesis, expression profiling and biological functions. Insect Biochem Mol Biol 43: 24-38, 2013.

25. Jung HJ and Suh Y: MicroRNA in aging: From discovery to biology. Curr Genomics 13: 548-557, 2012.

26. Banerjee J, Chan YC and Sen CK: MicroRNAs in skin and wound healing. Physiol Genomics 43: 543-556, 2011.

27. Krol J, Loedige I and Filipowicz W: The widespread regulation of microRNA biogenesis, function and decay. Nat Rev Genet 9: 597-610, 2010

28. Greussing R, Hackl M, Charoentong P, Pauck A, Monteforte R, Cavinato M, Hofer E, Scheideler M, Neuhaus M, Micutkova L, et al: Identification of microRNA-mRNA functional interactions in UVB-induced senescence of human diploid fibroblasts. BMC Genomics 14: 224, 2013.

29. Yi R and Fuchs E: MicroRNA-mediated control in the skin. Cell Death Differ 17: 229-235, 2010.

30. Hildebrand J, Rütze M, Walz N, Gallinat S, Wenck H, Deppert W, Grundhoff A and Knott A: A comprehensive analysis of microRNA expression during human keratinocyte differentiation in vitro and in vivo. J Invest Dermatol 13: 20-29, 2011.

31. Livak KJ and Schmittgen TD: Analysis of relative gene expression data using real-time quantitative PCR and the 2(-Delta Delta C(T)) method. Methods 25: 402-408, 2001.

32. Matsuzaki Y, Koyama M, Hitomi T, Yokota T, Kawanaka M, Nishikawa A, Germain D and Sakai T: Arctiin induces cell growth inhibition through the down-regulation of cyclin D1 expression. Oncol Rep 19: 721-727, 2008.

33. Hayashi K, Narutaki K, Nagaoka Y, Hayashi T and Uesato S: Therapeutic effect of arctiin and arctigenin in immunocompetent and immunocompromised mice infected with influenza A virus. Biol Pharm Bull 33: 1199-1205, 2010.

34. Binic I, Lazarevic V, Ljubenovic M, Mojsa J and Sokolovic D: Skin ageing: Natural weapons and strategies. Evid Based Complement Alternat Med 2013: 827248, 2013.

35. Baohua Y and Li L: Effects of SIRT6 silencing on collagen metabolism in human dermal fibroblasts. Cell Biol Int 36: 105-108, 2012.

36. Tilstra JS, Clauson CL, Niedernhofer LJ and Robbins PD: NF- $\kappa B$ in aging and disease. Aging Dis 2: 449-465, 2011.

37. Osorio FG, López-Otín C and Freije JM: NF-kB in premature aging. Aging (Albany NY) 4: 726-727, 2012.

38. Tanaka K, Asamitsu K, Uranishi H, Iddamalgoda A, Ito K, Kojima $\mathrm{H}$ and Okamoto T: Protecting skin photoaging by NF-kappaB inhibitor. Curr Drug Metab 11: 431-435, 2010. 